\title{
Telepsychiatric Consultation as a Training and Workforce Development Strategy for Rural Primary Care
}

\author{
Morbaf Al Acbkar, MD, $P b D^{1}$ \\ Ian M. Bennett, $M D, P b D^{1,2}$ \\ Lydia Chwastiak, MD, MPH ${ }^{2}$ \\ Theresa Hoeft, $\mathrm{PbD}^{2}$ \\ Tre Normoyle, $\mathrm{PbD}^{3}$ \\ Melinda Vredevoogd, $M S^{2}$ \\ Davis G. Patterson, $\mathrm{PbD}^{1}$ \\ ${ }^{1}$ Department of Family Medicine, Univer- \\ sity of Washington, Seattle, Washington \\ ${ }^{2}$ Department of Psychiatry and Behav- \\ ioral Sciences, University of Washington, \\ Seattle, Washington \\ ${ }^{3}$ Valley View Health Center, Chehalis, \\ Washington
}

\section{in \\ MORE ONLINE \\ www.annfammed.org}

AC Annals Journal Club selection; see inside back cover or https://www.Ann FamMed.org/content/AJC/..

Conflicts of interest: authors report none.

\section{CORRESPONDING AUTHOR}

Morhaf Al Achkar, MD, PhD Department of Family Medicine University of Washington 314 NE Thornton Place Seattle, WA 98125 alachkar@uw.edu

\begin{abstract}
PURPOSE There is a shortage of rural primary care personnel with expertise in team care for patients with common mental disorders. Building the workforce for this population is a national priority. We investigated the feasibility of regular systematic case reviews through telepsychiatric consultation, within collaborative care for depression, as a continuous training and workforce development strategy in rural clinics.
\end{abstract}

METHODS We developed and pilot-tested a qualitative interview guide based on a conceptual model of training and learning. We conducted individual semistructured interviews in 2018 with diverse clinical and nonclinical staff at 3 rural primary care sites in Washington state that used ongoing collaborative care and telepsychiatric consultation. Two qualitative researchers independently analyzed transcripts with iterative input from other research team members.

RESULTS A total of 17 clinical, support, and administrative staff completed interviews. Participants' feedback supported the view that telepsychiatric case reviewbased consultation enhanced skills of diverse clinical team members over time, even those who had not directly participated in case reviews. All interviewees identified specific ways in which the consultations improved their capacity to identify and treat psychiatric disorders. Perceived benefits in implementation and sustainability included fidelity of the care process, team resilience despite member turnover, and enhanced capacity to use quality improvement methods.

CONCLUSIONS Weekly systematic case reviews using telepsychiatric consultation served both as a model for patient care and as a training and workforce development strategy in rural primary care sites delivering collaborative care. These are important benefits to consider in implementing the collaborative care model of behavioral health integration.

Ann Fam Med 2020;18:438-445. https://doi.org/10.1370/afm.2561.

\section{INTRODUCTION}

$\longrightarrow$ ommon mental health disorders, including depression and anxiety, are leading causes of global disability ${ }^{1}$ and contribute substantially to premature mortality., Most treatment of behavioral health disorders occurs in primary care, which has been described as the de facto mental health system of the United States. ${ }^{3}$ This association is particularly true in rural areas, where the burden of these disorders (and suicide) is greater and specialty services are less available than in urban settings. ${ }^{4-6}$ Building capacity of rural primary care clinics to deliver high-quality mental health care, including through workforce development, is a major goal of national health policy.7.8 Behavioral health integration models, which provide specialty care input within primary care settings, are widely seen as a means of enhancing mental health care capacity, but are often insufficient to meet demand because of a limited workforce, especially in rural settings. ${ }^{9}$ Developing clinical skills of existing rural clinicians and staff could rapidly and sustainably build capacity to address community mental health needs. ${ }^{10}$

The collaborative care model of integrated care has a large evidence base supporting its effectiveness in treating behavioral health disorders 
in primary care. National policy efforts and new billing codes for bundled payment have increased the model's dissemination, but this complex model requires substantial effort to implement. ${ }^{11-14}$ In this integrated care model, primary care physicians retain primary responsibility for treatment of behavioral health disorders with the support of 2 new team members: a behavioral health care manager and a consulting psychiatrist (Figure 1). Care managers (typically social workers, therapists, or nurses) work in the primary care clinic, provide measurement-based care and care coordination, and also may offer brief psychotherapy for depression or anxiety. Consulting psychiatrists

\section{Figure 1. Initial conceptual model for training support by weekly systematic case review through telepsychiatry consultation.}

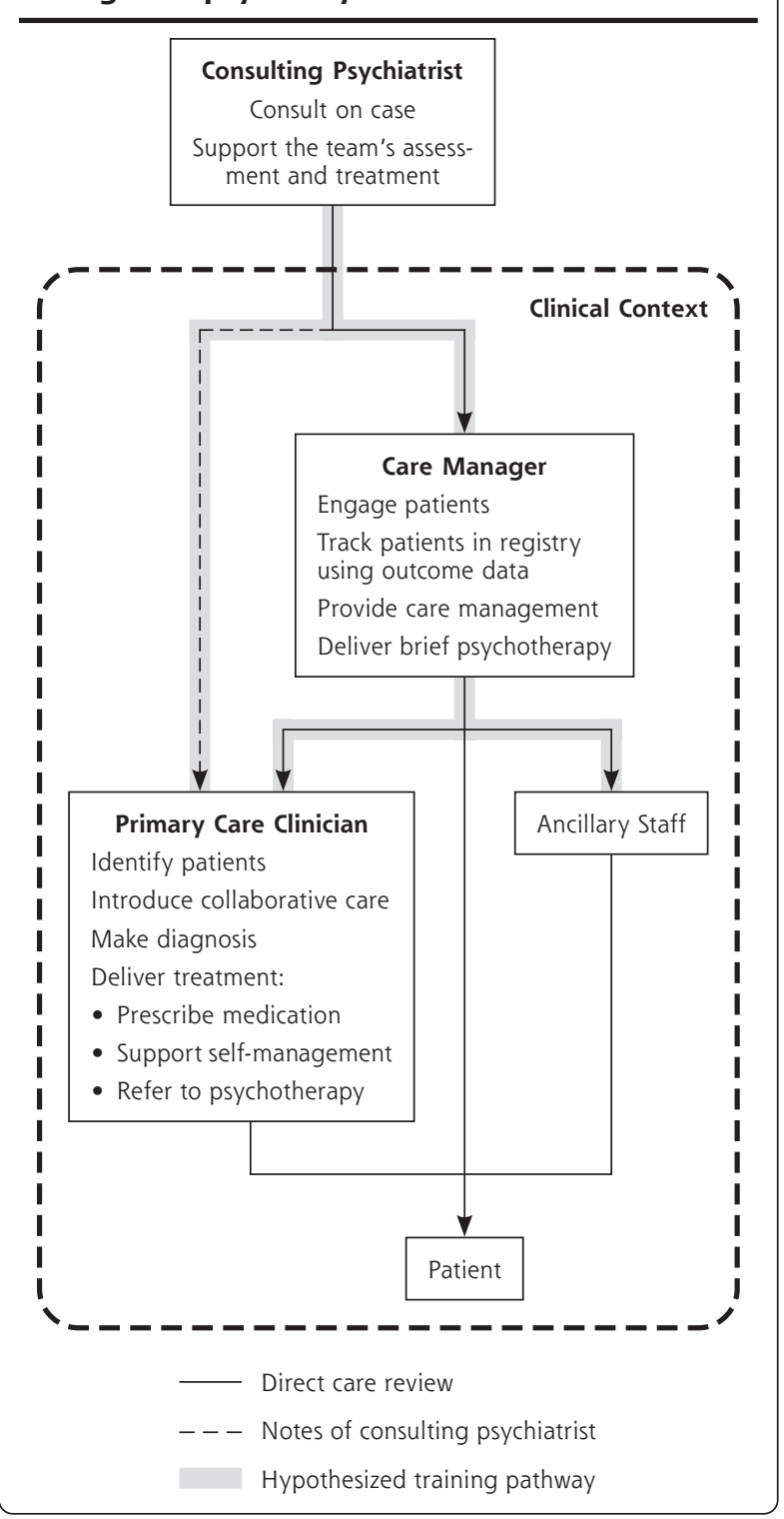

provide expert recommendations on patient care through weekly caseload reviews with care managers, using tools and workflows guided by principles of effective chronic disease management, including a registry for tracking outcomes and process measures, measurement-based care, evidence-based treatments, a treatment-to-target strategy, and stepped care. ${ }^{15-17}$ During regular systematic case reviews, psychiatrists provide input on patients through in-person or remote teleconsultation, leveraging psychiatric expertise to deliver specialty mental health care to more patients. ${ }^{18}$

An important principle of traditional specialty consultation is to disseminate authoritative clinical knowledge to the referring clinician and medical community. ${ }^{19-22}$ This educational function improves the treatment of the patient for whom consultation is sought and enhances the care and outcomes of future patients with similar clinical presentations. Regular systematic case review may provide contextualized training that enhances a range of patient, clinician, and implementation and sustainability outcomes. ${ }^{23} \mathrm{~A}$ clinical team's continuous learning via input on patient outcomes and evidence-based treatment options is a key element of a learning health care system. ${ }^{24}$ We hypothesized that over time, the collaborative care model serves as a training and workforce development strategy, a potentially important additional benefit. This qualitative study examined whether the model supports knowledge transmission (eg, training and workforce development) in rural primary care settings.

\section{METHODS}

We conducted a qualitative study using semistructured interviews. One qualitative investigator completed interviews from November 2016 through April 2017 at 3 rural or rural-serving primary care sites in Washington state that had implemented the collaborative care model in community health centers through the state's Mental Health Integration Program. Sites with successful implementation-based on sustained high rates of clinical improvement-were selected for the study. We followed a purposeful sampling strategy to include representation of all team member roles at 2 sites: clinicians, support personnel, and administrative staff. At both sites, clinicians and staff had received formal training in the collaborative care model, and the sites had provided this model for at least 2 years.

The formal training elements were part of a standard implementation approach of the Advancing Integrated Mental Health Solutions (AIMS) Center of the University of Washington, described in more detail elsewhere..$^{25}$ Briefly, formal training included a 1-day workshop for collaborative care model team members, 
primary care clinicians, and the internal implementation team and a second day of skills-based training for the care managers. In addition, a series of webinars on training topics were available for all clinicians and staff. Psychiatrists led the training, along with behavioral health leads if present at the clinic. Behavioral health leads had administrative responsibility for the collaborative care model along with other behavioral health responsibilities. They allocated time to train care team members and coordinate communication among organizational leadership, care team members, and other staff. These individuals sometimes also had duties as care managers and worked with patients directly.

We developed a conceptual model of training through telepsychiatric consultation based on team expertise and literature on educational learning models, including consultation, informal learning, and just-in-time learning (Figure 1). ${ }^{26-29}$ We conceptualized consultation within an implementation science framework, in which consultants have both specific expertise in intervention strategies as well as knowledge and experience of applying these strategies in varied settings. ${ }^{26}$ We distinguished between informal and formal learning. Formal learning involves removing the learner from day-to-day work to join in simulations, lectures, and other instructional activities. Informal learning, in contrast, is mainly a social process that occurs through action and reflection involving coaching and mentoring, self-directed learning, networking, and trial and error. ${ }^{27}$ Just-in-time training provides information at predetermined intervals, ensuring effective and timely dissemination of critical information to learners. ${ }^{28,29}$

We used our conceptual model to develop tailored guides for interviewing collaborative care model team members in different roles (consulting psychiatrist, care manager, primary care clinician, and medical staff). We pilot-tested the interview guides with collaborative care model team members in a university hospital-based clinic and revised the guides based on their feedback (the final version is included in Supplemental Appendix 1, https://www.AnnFamMed.org/ content/18/5/438/suppl/DC1/).

The first qualitative investigator conducted interviews in person during site visits, except for 3 primary care physicians who were interviewed by telephone. Interviews ranged from 21 to 95 minutes, averaging 56 minutes. We audio-recorded and transcribed all interviews. Two study team members independently reviewed transcripts for accuracy and removed identifying information before analysis with NVivo 11 Pro qualitative software (QSR International).

With the entire study team's input, the first qualitative investigator and a second qualitative investigator independently completed an iterative thematic analysis. First, we captured text describing interactions where training, learning, or both occurred. We then coded each excerpt using low-level inferences. ${ }^{30} \mathrm{We}$ further organized the codes according to interviewees' clinic roles. Finally, we grouped the codes under themes and subthemes. The study team met as a group to review and revise the iterative thematic analysis, during which our diverse backgrounds supported critical reflections.

Investigator positions and expertise were complementary. The first qualitative investigator, who led design and data collection, holds a $\mathrm{PhD}$ in sociology and is a health analyst focused on community engagement. The second qualitative investigator, who holds an $\mathrm{MD}$ and $\mathrm{PhD}$ and is trained in qualitative methodologies, brought sensitivity to education conceptualization frameworks. Other team members included a primary care physician, a psychiatrist, a health economist, and a sociologist and health services researcher with expertise in rural health and primary care. All team members had research or clinical experience with behavioral health integration into primary care. The University of Washington Institutional Review Board approved the study.

\section{RESULTS}

We completed interviews with 17 participants working in rural clinics that used collaborative care teams with telepsychiatry consultation. Table 1 presents their characteristics. Participants had a median of 2 years of experience in collaborative care.

\begin{tabular}{|c|c|}
\hline Characteristic & Value \\
\hline \multicolumn{2}{|l|}{ Team role, No. } \\
\hline Primary care clinician & 4 \\
\hline Care manager & 9 \\
\hline Consulting psychiatrist & 3 \\
\hline Medical assistant & 1 \\
\hline \multicolumn{2}{|l|}{ Age group, No. } \\
\hline $20-29$ y & 1 \\
\hline $30-39$ y & 5 \\
\hline $40-49$ y & 2 \\
\hline $50-59 y$ & 6 \\
\hline$\geq 60$ y & 3 \\
\hline \multicolumn{2}{|l|}{ Sex (self-identified), No. } \\
\hline Female & 10 \\
\hline Male & 7 \\
\hline \multicolumn{2}{|c|}{ Years of experience, median (range) } \\
\hline Since finishing training & $7(1-39)$ \\
\hline In collaborative care & $2(0.25-10)$ \\
\hline
\end{tabular}




\section{Learning and Training Themes}

We identified a total of 17 themes related to team members' training and learning in the collaborative care model. We discuss these themes categorized by team member role.

\section{Primary Care Clinicians}

In the collaborative care model, primary care clinicians learned to work proficiently with patients with mental illness. We identified 4 themes related to primary care clinician training and learning, outlined in Supplemental Table 1 (https://www.AnnFamMed.org/ content/18/5/438/suppl/DC1/) and discussed below.

First, primary care clinicians taking jobs in these rural clinics often had little behavioral health training and scant exposure to the collaborative care model. With minimal training or experience-many were in their first job-primary care clinicians in these rural clinics were often initially unprepared and uncomfortable caring for patients with mental illnesses. They were also rarely aware of community behavioral health resources and had minimal exposure to, and no formal training in, the collaborative care model.

Second, through the collaborative care model, primary care clinicians became more competent diagnosing mental illnesses and recognizing the importance of mental health. They learned to identify the presentation of behavioral health disorders and diagnostic criteria. They also gained knowledge of differential diagnosis and the impact of alcohol and substance use on mental health.

Third, primary care clinicians developed appropriate language for engaging patients and competency to formulate treatment plans. By listening to the care manager and reading the psychiatrist's notes on previous patients, primary care clinicians acquired language for talking to patients about mental illness and introducing them to treatment options. For example, some teams referred to care managers as life coaches or health coaches to help engage individuals who might resist seeing a psychiatrist or therapist. Primary care clinicians learned about appropriate medication regimens from interactions with the psychiatrist. They also developed an appreciation for nonpharmacologic behavioral health interventions and management strategies. With the care manager's on-site availability, they began connecting patients to behavioral health counseling services more.

Fourth, through participating in the collaborative care model, primary care clinicians became more proficient in managing behavioral health disorders, which transferred to the care of other patients with behavioral health needs. They became more comfortable making decisions and managing behavioral health disorders, and expanded their treatment options. The instruction and knowledge they received were then applied to additional patients. Primary care clinicians grew professionally as their experience increased. Their prescribing practices also improved, even with more complex mental health conditions, and they became more autonomous. By relying more on validated screening and measurement tools, participants reported the quality of care improved.

\section{Care Managers}

Care managers integrated an evidence-based model for mental health in primary care. We identified 5 themes related to care manager training and learning, outlined in Supplemental Table 2 (https://www.AnnFamMed. org/content/18/5/438/suppl/DC1/) and discussed below.

First, care managers were initially unprepared for the collaborative care model. Many reported never having worked or received training in the collaborative care model. Some also had limited knowledge of the presentation of behavioral health disorders. Over time, they developed skills through regular caseload reviews with the consulting psychiatrist and contextualized interactions with primary care clinicians and patients.

Second, care managers learned the biopsychosocial model of patient care within an integrative framework. After becoming familiar with this model, care managers grew to appreciate how medical issues affect mental health. They changed their style of interaction with patients to adapt to the faster pace of primary care as compared with that in mental health specialty settings.

Third, care managers developed new competencies in building relationships and working effectively on a care team. They learned to work with primary care clinicians, respecting their busy schedules, diverse work styles, and communication preferences. By being mindful of primary care clinicians' preferences, they learned how to build relationships and tailor their approach to each clinician. They also received coaching from the psychiatrist to effectively present recommendations to primary care clinicians.

Fourth, care managers increased their proficiency in diagnosing and managing mental illnesses. Care managers learned systematic approaches to assessment and diagnosis of mental illnesses, including differential diagnosis. They learned about medications and evaluation of response to treatment. Care managers also received coaching on indications for and delivery of brief behavioral interventions.

Fifth, care managers expanded their competency and improved quality of their care by applying learning to the care of new patients. Psychiatrists provided a scaffold for care managers' professional growth. Beyond the impact on new patients, the care managers 
became more prepared for new situations. Care quality reportedly improved over time, especially through use of evidence-based tools for screening and evaluation of treatment responses.

\section{Consulting Psychiatrists}

In the collaborative care model, consulting psychiatrists learned to coach the team and to lead program implementation. We identified 4 themes related to the consulting psychiatrists' learning, outlined in Supplemental Table 3, (https://www.AnnFamMed.org/ content/18/5/438/suppl/DC1/) and discussed below.

First, psychiatrists gained new perspectives and insights. They learned how the collaborative care model differs from traditional consult-liaison psychiatry. They also learned about primary care culture, the local community, and (often limited) treatment referral resources.

Second, psychiatrists played an educator role. They learned to become model teachers and taught by expounding clinical decision making. Although often directive in pursuing efficiency, the consulting psychiatrist also engaged learners in rich conversations as an educational strategy.

Third, psychiatrists came to appreciate that team learning is bidirectional. They learned to approach primary care team members with humility, knowing that primary care clinicians and care managers are also experts with valuable information relevant to patient care. Care managers served as sources of information for patients and clinicians that was critical for effective consultation. Psychiatrists also learned to leverage team members' expertise and creativity.

Fourth, psychiatrists, with their teams, learned the requirements for a sustainable program. They determined that continued efforts were needed to maintain program components and fidelity, as sustaining the collaborative care model requires building relationships with clinicians and engaging leadership. Finally, psychiatrists learned the importance of retraining team members in the face of high staff and clinician turnover.

\section{Ancillary Staff}

Through the model, ancillary staff learned to support collaborative care model tasks. We identified 4 themes related to ancillary staff learning, outlined in Supplemental Table 4 (https://www.AnnFamMed.org/ content/18/5/438/suppl/DC1/) and discussed below.

First, medical assistants learned to screen for mental illness and talk to affected patients. With care manager coaching, medical assistants developed competencies relevant to caring for this population. Over time, they came to understand mental illness as an important problem and became more active in collaborative care model roles.
Second, interpreters learned how to work in a medical setting using the collaborative care model. Because these rural clinics served large populations of Spanishspeaking patients, training the interpreters became essential for the model's implementation.

Third, receptionists learned how to schedule behavioral health appointments for care managers. They also learned to communicate with patients about visit scheduling and duration, which sometimes needed to be longer.

Fourth, billers became more proficient at billing for behavioral health services. Over time, they learned to support the collaborative care model by applying appropriate billing codes for care management services, which helped to provide sustainable revenue.

\section{Final Model}

Collectively, the above findings prompted us to revise our initial model of knowledge transmission and training to a final model, shown in Figure 2. Key elements include a range of formal and informal learning processes, as well as much more complex information flows, including bidirectionally with the consulting psychiatrist. Learning pathways for primary care clinicians and care managers are presented in Supplemental Appendixes 2 and 3 (https://www.AnnFamMed.org/ content/18/5/438/suppl/DC1/). We identified consistent evidence that informal learning occurred regularly through interaction of diverse clinical team members and was associated with the case review process. Additional implementation and sustainability themes included support for the fidelity of care processes, attention to quality improvement, and resilience to staff turnover.

\section{DISCUSSION}

Our study of rural clinics with established collaborative care model programs provides evidence that this model serves as a training and workforce development strategy. Although formal training of the collaborative care model team was necessary for successful implementation, after the initial program launch, all team members continued learning from each other through their interactions. Learning went beyond just-in-time information, coaching, and support from the consulting psychiatrist. Treatment of individual patients led to accumulation of experience with screening, assessment, and treatment strategies that could be applied to other patients.

All study participants perceived improvements in their competency as part of a chronic care team and provided clear examples of their own and other team members' enhanced patient interactions and 
proficiency in care delivery. These distinct personnel would not otherwise have worked together on this particular issue, and thus, their individual and team learning (depicted in Figure 2) supported implementation and sustainability of ongoing care using the collaborative care model. ${ }^{23}$ Furthermore, these interactions supported the transformation of existing clinic personnel into an effective and resilient chronic care team.

Figure 2. Final model of learning and training pathways and content for telepsychiatry consultation collaborative care.

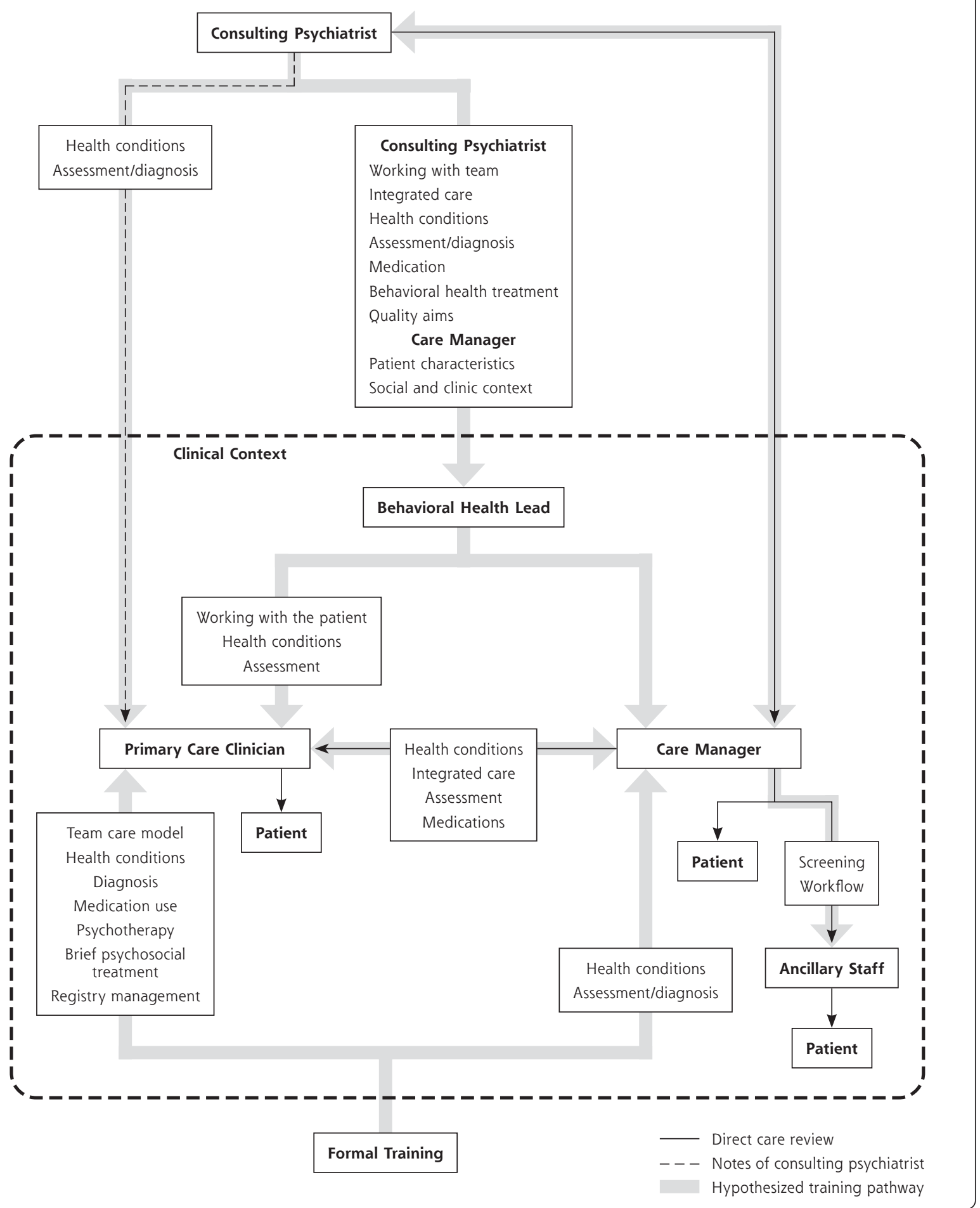

ANNALS OF FAMILY MEDICINE + WWW.ANNFAMMED.ORG + VOL. 18, NO. 5 + SEPTEMBER/OCTOBER 2020 
Policy makers interested in rural mental health and development of a chronic illness workforce should consider this specialist consultation model of team care a promising framework for rapidly building existing primary care capacity to improve care outcomes.

Training consulting psychiatrists and care managers to focus explicitly on the model's workforce development benefits may reduce variability in integrated behavioral health and maximize the potential to achieve the goal of high-quality care for all rural patients. Furthermore, to be perceived as effective educators, consulting psychiatrists needed to learn about the team's work culture and the rural communities they served. These consultants taught by describing their thought processes and making explicit recommendations that team members could apply to future patient encounters. They were also perceived as partners and treated the team with humility, recognizing that learning was bidirectional. These notions can be incorporated into training of psychiatrists for their future role in the collaborative care model.

Several lines of evidence support our conclusion that the collaborative care model provided continuous learning at study sites. First, all participants reported having little experience with mental health and teambased care before model implementation. Second, documented learning included aspects unique to the collaborative care model, such as improving population health, using evidence-based treatments, and communicating as a team. Third, participants reported convergence between learners' practices and those of more knowledgeable team members. Examples of these convergences included reasoning for medication prescribing, the language of patient engagement, and evidence-based behavioral interventions. Lastly, team care members often named precisely where in the care process and how they learned specific practices. For example, primary care clinicians reported learning how to present the collaborative care process to patients from care managers, care managers described learning how to carry out mental health assessments from consulting psychiatrists, and psychiatrists described learning from care managers about patient needs and available community resources.

The concept of a learning health care system frames key elements that drive improvements in quality of care including the use of clinical teams to work with patients to tailor care to their specific needs. ${ }^{24}$ Central to this framework is monitoring care delivery and patient outcomes for continuous learning, facilitated by health informatics tools. Although no universal approach exists for achieving these goals, our study highlights the feasibility of the collaborative care model for treating common mental disorders.
This study brings important new knowledge to the field of behavioral health integration, but we also acknowledge limitations. First, we studied a small number of sites whose attributes may not generalize to others. These sites represent the perspectives of mature, high-functioning collaborative care model exemplars. This selection was appropriate for our goal of understanding information flow in sites that have effectively implemented this complex intervention. Our in-depth explorations of diverse personnel allowed for better understanding of learning and discerning lessons that can be shared in other rural primary care settings that are considering adopting the collaborative care model. Additional investigation of less successful sites could provide valuable insights about a wider range of clinics. Second, we were unable to assess objective evidence of learning flows through clinical teams that would have helped gauge the relative importance of the themes we identified. Nevertheless, triangulating the perceptions of individuals occupying different roles yielded consistent evidence of team learning. Finally, this study focused on the collaborative care model, and conclusions about continuous learning may not apply to other integrated care models.

To our knowledge, this is the first study exploring whether the collaborative care model serves not only as an evidence-based model of patient care, but also as a training and workforce development strategy within primary care sites. Although robust evidence supports the collaborative care model's clinical benefit and national policies support its implementation, these additional benefits should be considered when choosing among distinct models of behavioral health integration for local implementation. ${ }^{31}$ High-performing collaborative care model programs show evidence of continuous learning, but this model's implementation is complex and highly variable. ${ }^{14}$ Additional research should investigate how this variability may relate to patient outcomes and assess variability in elements of training and workforce development. Research is also needed to determine how the collaborative care model's continuous learning aspects might address common challenges, such as staff turnover, and determine the extent to which our findings can support behavioral health integration in rural settings.

To read or post commentaries in response to this article, see it online at https://www. AnnFamMed.org/content/18/5/438.

Submitted July 3, 2019; submitted, revised, February 25, 2020; accepted March 9, 2020.

Key words: rural health services; mental health; telepsychiatry; remote consultation; telemedicine; consultation; collaborative care; primary care; depression; patient care team; vulnerable populations

Funding support: Research supported by the Bureau of Health Workforce (BHW), Health Resources and Services Administration (HRSA), U.S. 
Department of Health and Human Services (HHS) under cooperative agreement UH1HP29966.

Disclaimer: Information, conclusions, \& opinions expressed are of the authors, and no endorsement is intended or should be inferred.

Prior presentations: An earlier version of this work was presented at the 2017 North American Primary Care Research Group annual conference; November 17-21, 2017; Montreal, Quebec, Canada.

Acknowledgments: Our thanks to the clinical teams that participated in the research presented. We appreciate the expert support of Johnny Mao and others in early stages of this work and to Tess Grover for her excellent support in the preparation of figures. We very much appreciate the AIMS Center of the University of Washington for support in identifying sites to participate in the study as well as connections with the psychiatric consultants from the Department of Psychiatry and Behavioral Sciences. Finally, particular thanks to Dr. Jürgen Unützer for support of this work.

Author contributions: M.A. contributed significantly to the analytic framework and led the qualitative analysis; led the write-up of the results and methods; and made a substantial contribution to the discussion and led the write-up and revisions. I.M.B. conceptualized the research and led the design of the study; supervised data collection; contributed to interpreting the results; and made a substantial contribution to the literature review and the write-up of the background and discussion. L.C. contributed to the design of the study and the interpretation of results, and also made a substantial contribution to the revision. T.H. contributed to the qualitative analysis and interpretation of results, and also contributed to the writing of all study sections. M.V. contributed to data acquisition and empirical methods, and also contributed to the writing of various sections. T.N. contributed to data acquisition. D.G.P. oversaw all phases of the project and contributed study design, analysis, and writing. All authors reviewed and approved the final draft.

Supplementary materials: Available at https://www.AnnFam Med.org/content/18/5/438/suppl/DC1/.

\section{References}

1. Ferrari AJ, Norman RE, Freedman G, et al. The burden attributable to mental and substance use disorders as risk factors for suicide: findings from the Global Burden of Disease Study 2010. PLoS One. 2014;9(4):e91936.

2. Murray CJ, Vos T, Lozano R, et al. Disability-adjusted life years (DALYs) for 291 diseases and injuries in 21 regions, 1990-2010: a systematic analysis for the Global Burden of Disease Study 2010. Lancet. 2012;380(9859):2197-2223.

3. Regier DA, Narrow WE, Rae DS, Manderscheid RW, Locke BZ, Goodwin FK. The de facto US mental and addictive disorders service system. Epidemiologic catchment area prospective 1-year prevalence rates of disorders and services. Arch Gen Psychiatry. 1993;50(2):85-94.

4. Rossen LM, Hedegaard $H$, Khan D, Warner M. County-level trends in suicide rates in the US, 2005-2015. Am J Prev Med. 2018;55(1):72-79.

5. Thomas D, Macdowell M, Glasser M. Rural mental health workforce needs assessment-a national survey. Rural Remote Health. 2012; 12(4):2176.

6. Dewan M, Meszaros ZS, Manring J. Addressing the shortage of psychiatrists in rural areas. Psychiatr Serv. 2014;65(12):1508.

7. Goodwin G, Tobler L. Improving rural health: State policy options. Washington, DC: National Conference of State Legislatures. https:// bit.ly/32MujAx. Published 2013. Accessed Apr 7, 2019.

8. US Department of Health $\&$ Human Services. Strategic goal 1: Reform, strengthen, and modernize the nation's healthcare system. https://bit.ly/3bhIX6M. Accessed Apr 7, 2019.
9. Skillman SM, Snyder CR, Frogner BK, Patterson DG. The Behavioral Health Workforce Needed for Integration With Primary Care: Information for Health Workforce Planning. Seattle, WA: Center for Health Workforce Studies, University of Washington; 2016.

10. Kroenke K, Unutzer J. Closing the false divide: sustainable approaches to integrating mental health services into primary care. J Gen Intern Med. 2017;32(4):404-410.

11. Gilbody S, Bower P, Fletcher J, Richards D, Sutton AJ. Collaborative care for depression: a cumulative meta-analysis and review of longer-term outcomes. Arch Intern Med. 2006;166(21):2314-2321.

12. Williams D, Eckstrom J, Avery M, Unützer J. Perspectives of behavioral health clinicians in a rural integrated primary care/mental health program. J Rural Health. 2015;31(4):346-353.

13. Carlo $A D$, Jeng PJ, Bao Y, Unützer J. The learning curve after implementation of collaborative care in a state mental health integration program. Psychiatr Serv. 2019;70(2):139-142.

14. Katon W, Unützer J. Collaborative care models for depression: time to move from evidence to practice. Arch Intern Med. 2006;166(21): 2304-2306.

15. Von Korff M, Tiemens B. Individualized stepped care of chronic illness. West J Med. 2000;172(2):133-137.

16. Unützer J, Park M. Strategies to improve the management of depression in primary care. Prim Care. 2012;39(2):415-431.

17. Trivedi $\mathrm{MH}$. Treating depression to full remission. J Clin Psychiatry. 2009;70(1):e01.

18. Fortney JC, Pyne JM, Turner EE, et al. Telepsychiatry integration of mental health services into rural primary care settings. Int Rev Psychiatry. 2015;27(6):525-539.

19. Hilty DM, Yellowlees PM, Cobb HC, Bourgeois JA, Neufeld JD, Nesbitt TS. Models of telepsychiatric consultation-liaison service to rural primary care. Psychosomatics. 2006;47(2):152-157.

20. Pearson SD. Principles of generalist-specialist relationships. J Gen Intern Med. 1999;14(Suppl 1):S13-S20.

21. Goldman L, Lee T, Rudd P. Ten commandments for effective consultations. Arch Intern Med. 1983;143(9):1753-1755.

22. Salerno SM, Hurst FP, Halvorson S, Mercado DL. Principles of effective consultation: an update for the 21st-century consultant. Arch Intern Med. 2007;167(3):271-275.

23. Proctor $E$, Silmere $H$, Raghavan R, et al. Outcomes for implementation research: conceptual distinctions, measurement challenges, and research agenda. Adm Policy Ment Health. 2011;38(2):65-76.

24. McGinnis JM, Stuckhardt L, Saunders R, Smith M, eds. Best Care at Lower Cost: The Path to Continuously Learning Health Care in America. Washington, DC: National Academies Press; 2013.

25. Bowen DJ, Powers DM, Russo J, et al. Implementing collaborative care to reduce depression for rural native American/Alaska native people. BMC Health Services Research. 2020;20(1):34.

26. Nadeem E, Gleacher A, Beidas RS. Consultation as an implementation strategy for evidence-based practices across multiple contexts: unpacking the black box. Adm Policy Ment Health. 2013;40(6): 439-450.

27. Enos MD, Kehrhahn MT, Bell A. Informal learning and the transfer of learning: how managers develop proficiency. Hum Resour Dev Q. 2003;14(4):369-387.

28. Bolton MK. The role of coaching in student teams: A "just-in-time" approach to learning. J Manage Educ. 1999;23(3):233-250.

29. Hall L. Just-in-time learning: web-based/internet delivered instruction. AMCIS 1999 Proc. 1999:319.

30. Carspecken PF. Critical Ethnography in Educational Research: A Theoretical and Practical Guide. New York, NY: Routledge; 1994.

31. Press MJ, Howe R, Schoenbaum M, et al. Medicare payment for behavioral health integration. N Engl J Med. 2017;376(5):405-407. 